\title{
Association between Diet Quality and Perceived Stress in Women of Reproductive Age from a University Population in UK
}

\author{
K. Khaled ${ }^{1}$, V. Hundley ${ }^{2}$ and F. Tsofliou ${ }^{1,2}$ \\ ${ }^{1}$ Department of Rehabilitation \& Sport Sciences, Faculty of Health \& Social Sciences, Bournemouth University, \\ Bournemouth, UK and \\ ${ }^{2}$ Centre for Midwifery, Maternal \& Perinatal Health, Faculty of Health \& Social Sciences, Bournemouth University, \\ Bournemouth, UK
}

Diet quality of women of reproductive age is a major determinant of their weight status and health during pregnancy ${ }^{(1)}$. Obesity during pregnancy exhibits deleterious consequences on the mother and the offspring ${ }^{(1)}$. Stress has been linked with low diet quality, but no study assessed this link in women of reproductive age ${ }^{(2)}$. The study aimed to investigate the association between stress and diet quality in women of reproductive age in the UK.

An online questionnaire survey examining the correlation between stress and diet among 244 women of reproductive age from UK. Dietary assessment was explored via the validated European Prospective into Cancer and Nutrition food frequency questionnaire (EPIC FFQ). A-priori (hypothesis-driven) diet quality was derived from the EPIC FFQ by measuring the adherence to the Alternate Mediterranean Diet Index (aMD) which is based on 9 food components: whole grains, vegetables (without potatoes), fruits, alcoholic beverages, red/processed meats, nuts, fish, legumes, and the ratio of monounsaturated-fats to saturated-fats. Scores of aMD ranged from 0-9 where 0-3 indicated low adherence, 4-6 medium adherence, and 7-9 high adherence. A-posteriori (data-driven) dietary patterns were explored through applying factor analysis on 11 food groups (grams/day): fats and oils, sugars and snacks, cereals, alcoholic beverages, red/processed meats, fish and seafood, eggs, milk and milk products, fruits, vegetables, nuts and seeds. The survey measured stress through the 14-item Perceived Stress Scale (PSS). Adiposity measures were self-reported and physical Activity was estimated using the International Physical Activity Questionnaire (IPAQ). Additionally, the survey included socioeconomic questions. Factor analysis revealed three dietary patterns (DPs). Regression analysis was used to assess the predictors of each of the three DPs.

Overall, participants had a medium $(n=113)$ and/or low $(n=95)$ adherence to aMD; only few $(n=36)$ had a high adherence. Higher levels of stress were reported by participants with low adherence to aMD $(\mathrm{X} 2(2, \mathrm{~N}=244)=14.08, \mathrm{p}=0.001)$. Participants with high adherence to aMD tended to be of normal BMI $(\mathrm{p}=0.004)$. Using a minimum loading cut-off of 0.3 , factor analysis revealed three DPs: 1) "fats and oils, sugars and snacks, alcoholic beverages, red/processed meat, and cereals" DP (DP-1), 2) "fish and seafood, eggs, and milk and milk products" (DP-2), 3) "fruits, vegetables, nuts and seeds" (DP-3). Multiple regression models (Table 1) revealed that DP-1 was positively associated with stress $(p=0.005)$ and negatively with age $(p=0.004)$ and smoking $(p=0.005)$. DP-2 was found to be negatively associated with high maternal educational level $(p=0.01)$ while DP-3 was negatively associated with stress $(\mathrm{p}<0.001)$, BMI $(\mathrm{p}=0.001)$, white ethnicity $(\mathrm{p}=0.01)$.

Perceived stress was found to be negatively linked with diet quality and patterns among women of reproductive age in UK. Future well-designed trials are needed to investigate this relationship further.

\section{References}

1. Khaled K, Hundley V, Almilaji O, et al. (2020) Nutrients 12, 2921.

2. Khaled K, Tsofliou F, Hundley V, et al. (2020) Nutr J 19, 92. 